\title{
Water adsorption in hydrophobic MOF channels
}

\section{Selvarengan Paranthaman, François-Xavier Coudert* and Alain H. Fuchs}

\author{
Received 30th November 2009, Accepted 19th April 2010 \\ First published as an Advance Article on the web 7th June 2010 \\ DOI: $10.1039 / b 925074 c$
}

We report an investigation of water adsorption in the hydrophobic metal-organic framework $\mathrm{Al}(\mathrm{OH})(1,4-$ naphthalenedicarboxylate) by means of molecular simulation. We show how simple molecular models allow us to reproduce the experimental isotherm, and how grand canonical Monte Carlo simulations can help elucidate the question of the thermodynamic nature of the adsorption transition, which turns out to be a continuous transition, though the experimental isotherm is quite steep. Moreover, we study the influence of functionalisation of the MOF organic linkers on the hydrophobicity of the material and the nature of the adsorption transition, and explain it in terms of the liquid-vapour phase diagram of water in this family of materials.

\section{Introduction}

Nanoporous metal-organic frameworks (MOFs) are topical materials displaying a large range of crystal structures and host-guest properties, due to a combination of tunable porosity, by choice of metal centres and linker length, and functionalisation of the internal surface of the material. Among the proposed applications of MOFs, adsorptive storage and separation of strategic gases $\left(\mathrm{H}_{2}, \mathrm{CO}_{2}, \mathrm{CH}_{4}\right)$ are of particular importance and have gained a lot of attention in recent years. ${ }^{1,2}$ Though a very large number of materials with varying structures and chemistry have been synthesised, and the adsorption of a handful of gases is routinely performed along with structure characterisation, little data is available on water adsorption in MOFs. ${ }^{3-9}$ Some MOFs, such as MOF-5 (also known as IRMOF-1), HKUST-1 and DUT-4, are unstable in the presence of water vapour. ${ }^{10}$ Others, however, are stable even when left in liquid water for long periods of time. A coherent, global understanding of the factors behind the water stability of MOFs is still lacking and is hard to tackle; this is demonstrated, e.g., by the fact that materials of the same family such as $\mathrm{Al}(\mathrm{OH})(1,4-\mathrm{ndc})$ and $\mathrm{Al}(\mathrm{OH})(2,6-\mathrm{ndc})$ (ndc $=$ naphthalenedicarboxylate) can behave very differently, with the first material stable upon water adsorption ${ }^{11}$ while the second is not. ${ }^{3}$ Furthermore, even for MOFs that are stable upon water adsorption, few water adsorption (and desorption) isotherms have been published and the water adsorption phenomenon has not been widely investigated.

In contrast, water adsorption in other porous materials has been extensively studied, from both experimental and theoretical points of view. The behaviour of water confined in spaces of nanoscopic dimension is an important issue in many areas of science and technology. ${ }^{12,13}$ The special case where the confining matrix is hydrophobic, in particular, has attracted a lot of interest in the past decade. There are many systems for which water adsorption in hydrophobic spaces is

Chimie ParisTech, CNRS and Université Pierre et Marie Curie, 11 rue Pierre et Marie Curie, 75005 Paris, France.

E-mail: fx.coudert@chimie-paristech.fr; Fax: + 3314329 2059; Tel: +33144276751 relevant, including activated carbons and all-silica zeolites, ${ }^{14,15}$ functionalised or coated mesoporous materials ${ }^{16}$ as well as biological channels ${ }^{17,18}$ and protein cavities. ${ }^{19,20}$ Some simple questions remain yet to be answered, such as: for real-life, heterogeneous materials that can present hydrophilic defects, how does the water adsorption depend on the features of hydrophilic sites, such as their chemical nature, their concentration and their spatial distribution? These questions have been studied for model surfaces, ${ }^{21}$ adsorption on mixed self-assembled monolayers ${ }^{22}$ and in existing materials such as defective all-silica zeolites ${ }^{23,24}$ or zeolites with a very high $\mathrm{Si}$ : Al ratio (i.e., very low acidity). ${ }^{25}$ It seems thus only natural to extend these investigations to MOFs, which, by their very own chemical nature, present nanoscale heterogeneity.

In this article, we report a molecular simulation study of water adsorption in a rigid, stable MOF for which experimental adsorption and desorption water isotherms are available: $\mathrm{Al}(\mathrm{OH})(1,4-\mathrm{ndc})$. We show how simple molecular models can reproduce the observed type- $\mathrm{V}$ isotherm and shed some light on the nature of the adsorption transition. We also demonstrate the influence of pore size and functionalisation of the organic linker on the hydrophobicity of the material.

\section{Systems and computational methods}

\section{Systems studied: $\mathrm{Al}(\mathrm{OH})(1,4-n d c)$ and its derivatives}

We study in this article the adsorption of water in the $\mathrm{Al}(\mathrm{OH})(1,4-\mathrm{ndc})$ (ndc $=$ naphthalenedicarboxylate) metalorganic framework. This recent material was synthesized by Kitagawa et al., ${ }^{11}$ who also reported experimental adsorption isotherms for carbon dioxide, methanol, acetone, benzene and water. The crystal structure of the material is composed of parallel, 1D infinite $\mathrm{Al}(\mathrm{OH})$ chains along the $c$-axis, linked together in the other two directions by 1,4-naphthalenedicarboxylate ligands. This forms $1 \mathrm{D}$, parallel, noninterconnected square channels of two different types (see Fig. 1): small channels, into which the side aromatic ring of the 1,4-ndc is projecting, and larger channels, of size $7 \times 7 \AA^{2}$. The nanoporous framework is rigid and exhibits good thermal stability, both features being attributed to the $\pi-\pi$ interactions between aromatic rings. 


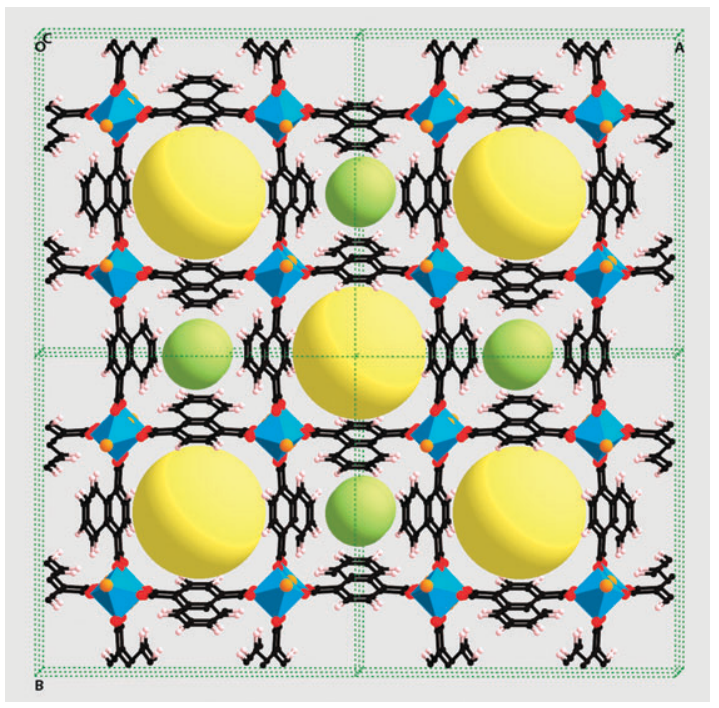

Fig. 1 View of a $2 \times 2 \times 2$ supercell of $\mathrm{Al}(\mathrm{OH})(1,4-\mathrm{ndc})$ along the $c$-axis. Aluminium coordination octahedra are represented in blue. For ease of visualisation of the 1D pores of the structure and their size, spheres are fitted inside them (yellow for large pores, green for small pores).

In the work presented herein, we have also studied the effect of pore size and MOF functionalisation by looking at the water adsorption in materials where some or all of the 1,4-ndc linkers are replaced by methyl-substituted derivatives: 6-methyl-1,4-ndc and 6,7-dimethyl-1,4-ndc. The additional methyl groups project into the larger channels, modulating the porosity of the material. Derivatives studied included 2, 4 and 8 methyl groups in the section of a large channel, over a total number of 8 possible substitution sites. The chosen distributions of the methylated carbon atoms are described in Fig. 2.

\section{Grand canonical Monte Carlo simulations}

The calculation of adsorption isotherms was performed using grand canonical Monte Carlo (GCMC) simulations to

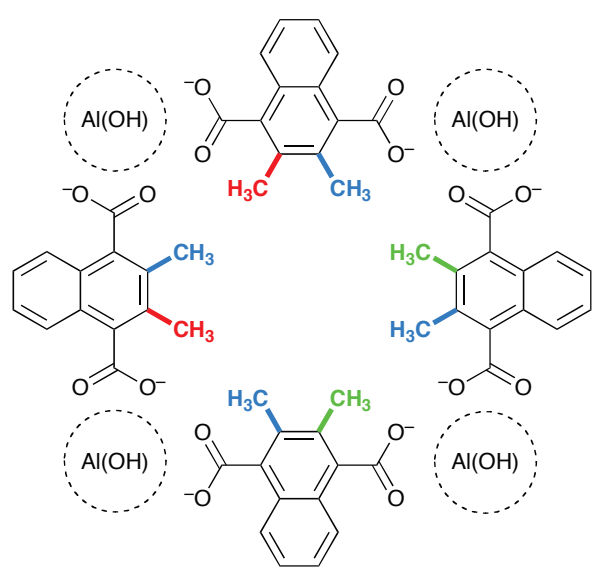

Fig. 2 Summary of the substitution sites used to create methylated derivatives of $\mathrm{Al}(\mathrm{OH})(1,4-\mathrm{ndc})$, in a schematic representation of a large square pore with $\mathrm{Al}(\mathrm{OH}) 1 \mathrm{D}$ pillars at the corners. The first two methyl groups introduced are represented in red, the next two in green and the last four are in blue. compute the average number of adsorbed water molecules per unit cell of the material, for a series of values of the chemical potential $\mu$. The chemical potential of water, $\mu$, was related to the thermodynamic pressure, $P$, by using the ideal gas law in the vapour phase (up to $P_{\text {sat }} \approx 5.4 \mathrm{kPa}$ for this model; the experimental value is $P_{\text {sat }} \approx 3.5 \mathrm{kPa}$ ), and by using the $\mu(P)$ relationship for TIP4P liquid water previously computed by Monte Carlo simulations. ${ }^{26}$ Pre-insertion, ${ }^{27}$ orientation $^{28,29}$ and displacement ${ }^{30}$ bias moves were used to accelerate the convergence of the GCMC simulations.

All Monte Carlo runs were performed on a $2 \times 2 \times 3$ supercell (of dimensions $42 \times 42 \times 20 \AA^{3}$ ) to minimise the influence of periodic boundary conditions; the dependence of results on system size is discussed later in this article. Each point of each isotherm was obtained after a first equilibration run of one billion $\left(10^{9}\right)$ steps, followed by a production run of another one billion steps for data collection. These unusually long simulation runs are made necessary by a very slow convergence of the system, which is linked to the fact that the one-dimensional pores are always totally filled or totally empty, with very slow switching between these two states (see the Results section).

In order to improve the efficiency of the calculations, both the electrostatic field created by the material and its repulsion-dispersion interaction energies with water were precomputed on a grid (with a mesh size of approximately $0.16 \AA$ ) and stored for use during the simulations.

\section{Density of states calculations}

In order to determine univocally the nature of the adsorption transitions in nanoporous materials, knowledge of the thermodynamic potential of the system is necessary. Such a quantity cannot be obtained by straightforward GCMC simulations, and requires the use of more sophisticated techniques. We have used here expanded ensemble density of states (EXEDOS) ${ }^{31,32}$ calculations to compute the grand potential of the system, which is the thermodynamic potential of the grand canonical ensemble $\Omega(\mu, N)$, as a function of chemical potential of the adsorbate, $\mu$, and number of adsorbed molecules, $N$. This method, developed by Wang and Landau, calculates the density of states of the system on the fly, using biased Monte Carlo simulations. ${ }^{33,34}$ It has been successfully used for a wide range of system, including Lennard-Jones fluids ${ }^{35}$ and simulations of adsorption in zeolites. ${ }^{23-25}$ For detailed about the implementation used here, the reader is referred to ref. 23 .

Density of states calculations were run on the same $2 \times 2 \times 3$ supercell as the GCMC simulations, for a total number of up to 6 billion steps (and a convergence factor of the density of states profile, or "flatness criterion", of 95\%). An OpenMPparallelised version of the simulation code running on 8 processor cores at a time was used for these calculations.

\section{Models and forcefields}

GCMC calculations of water adsorption inside $\mathrm{Al}(\mathrm{OH})(1,4-\mathrm{ndc})$ and its methylated derivatives were performed on frameworks considered rigid and modelled atomistically. Water molecules are described by the TIP4P-Ew model, ${ }^{36}$ a modified TIP4P 
potential for use with the Ewald summation technique, which was shown to present good thermodynamic properties for bulk water. ${ }^{37}$ Dispersion and repulsion interactions were modelled by $12-6$ Lennard-Jones potentials between all atoms, with a $10 \AA$ cutoff distance and long-range tail corrections applied. Lennard-Jones parameters for atoms of the framework were taken from the DREIDING forcefield, ${ }^{38}$ except for Al parameters which were taken from UFF. ${ }^{39}$ Lorentz-Berthelot mixing rules were used for calculation of water-MOF interactions.

Partial atomic charges for the material were adjusted in order to adequately describe the thermodynamics of water adsorption inside the nanopores of $\mathrm{Al}(\mathrm{OH})(1,4-\mathrm{ndc})$, and be transferrable between the functionalised materials, as described below. The Ewald summation technique was used to calculate all electrostatic interactions in the system.

\section{Results and discussion}

\section{Determination of partial charges for the MOF}

One of the key issues in molecular simulation studies of the adsorption of strongly polar molecules, such as water, in nanoporous materials, is the description of host-guest electrostatic interactions, which is typically performed using a nonpolarizable approximation of partial charges placed on the atoms of the system. Solving this complex issue and determining adequate charges for both the host and guest molecules is no small feat. Indeed, even though a great number of molecular models are available to describe bulk water, and some of them are known to describe quite reasonably the liquid-vapour phase envelope, the water molecules confined inside the nanopores of the material have different properties and should thus be described using a different model than for the bulk. One of the most striking differences between bulk and confined water was shown to be the difference in dipole moment, which is much smaller in the confined liquid due to the smaller extent of water-water polarisation. ${ }^{25,40}$ However, because it is much more practical to employ well-validated models for the adsorbate molecule than to redesign new potentials, it is quite common to use standard models for the adsorbate (validated for bulk behaviour) and to choose effective partial charges for the material that compensate these effects. This typically leads to charges that are smaller than the partial charges computed directly from quantum chemistry methods, whether they rely on some partitioning of the total electronic density between atoms (such as Mulliken or Löwdin analysis), or on fitting the electrostatic potential inside the nanopores. For example, this is well established for both simulations of gas-phase water adsorption and liquid-phase water intrusion in hydrophobic zeolites. ${ }^{14,41}$

Because the goal of this study is to probe the behaviour of water adsorption in a series of functionalised variants of the $\mathrm{Al}(\mathrm{OH})(1,4-\mathrm{ndc}) \mathrm{MOF}$, and also because little experimental thermodynamic data is available on this particular system, we used a very simple approach to adjust the partial atomic charges of the host material. We used as a starting point the partial charges determined by Calero et al. in a recent study of the related material MIL-47, ${ }^{42}$ in which benzenedicarboxylate ligands connect one-dimensional $\mathrm{V}(\mathrm{OH})$ chains to form lozenge-shaped pores. We then scaled all the partial charges down to better reproduce the experimental adsorption isotherm. While scaling down the charges, we placed an additional constraint: that the atomic charges of the atoms of the naphthalene rings, which was small in the original set, be exactly zero. This constraint leads to good transferability, allowing the same set of charges to be used for all methylsubstituted ligands.

The results obtained for the optimisation of the partial charges are consistent with those previously obtained for description of hydrophobic zeolites. When using the "standard" set of charges, the ionicity of the material is such that water adsorption takes place at very low pressure and follows a reversible type-I isotherm, ${ }^{43}$ which is definitely incoherent with the experimental isotherm. A type-I isotherm, which features a positive slope $\left(\mathrm{d} N_{\text {ads }} / \mathrm{d} P\right)$ in the low-pressure region, is typical of a wetting adsorbent-adsorbate pair, for which the interaction between the two are stronger than adsorbate-adsorbate interactions. We tried scaling the charges by $80,60,40,30$ and $20 \%$, and found that the set obtained with $30 \%$ scaling was consistent with the experimental data, i.e. its follows a type- $\mathrm{V}$ isotherm ${ }^{43}$ with adsorption and desorption taking place in the $0.6-1.2 \mathrm{kPa}$ range. Type- $\mathrm{V}$ isotherms are obtained for adsorption of nonwetting adsorbent-adsorbate pairs, where the adsorbent-adsorbate interaction is weak. The partial charges obtained at $30 \%$ scaling are reported in Table 1 . The experimental and simulated isotherms are shown together on Fig. 3 and are in reasonable agreement, taking into account the huge hysteresis loop of the experimental data, to which we cannot compare directly.

It should be noted that the adsorption-desorption behaviour of the system is very sensitive to small changes in the partial charges: with $q_{\mathrm{Al}}=+0.68(40 \%$ scaling $)$, we get an incorrect isotherm type, and with $q_{\mathrm{Al}}=+0.34$ (20\% scaling), the adsorption transition occurs in the liquid phase $\left(P>P_{\text {sat }}\right)$ instead of the gas phase. Indeed, the thermodynamics of adsorption of very polar molecules, such as water, in metal-organic frameworks makes a good touchstone for the development of robust, transferable MOF-adsorbate forcefields. In particular, it is striking to see how small the charges on the aluminium (formal charge: +3 ) and hydroxide group need to be to correctly reproduce the experimental water

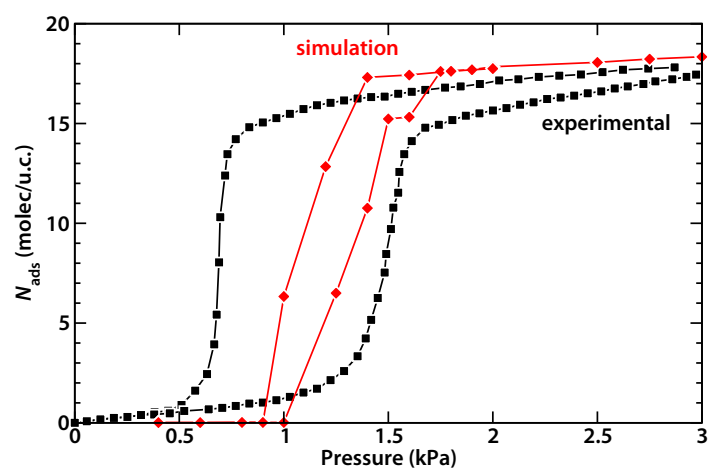

Fig. 3 Comparison of the experimental and simulated adsorption and desorption isotherms for water in $\mathrm{Al}(\mathrm{OH})(1,4-\mathrm{ndc})$ at $298 \mathrm{~K}$. 
Table 1 Atomic partial charges and Lennard-Jones parameters

\begin{tabular}{llll}
\hline Atom type & $\varepsilon / k_{\mathrm{B}} / \mathrm{K}$ & $\sigma / \AA$ & $q$ \\
\hline $\mathrm{Al}$ & 156.1 & 4.39 & 0.504 \\
$\mathrm{C}$ & 47.86 & 3.47 & 0 \\
$\mathrm{C}_{\mathrm{c}}$ & 47.86 & 3.47 & 0.132 \\
$\mathrm{H}_{\mathrm{h}}$ & 7.65 & 2.85 & 0.036 \\
$\mathrm{H}$ & 7.65 & 2.85 & 0 \\
$\mathrm{O}_{\mathrm{h}}$ & 48.19 & 3.03 & -0.180 \\
$\mathrm{O}_{\mathrm{c}}$ & 48.19 & 3.03 & -0.156
\end{tabular}

Subscript " $h$ " is used for atoms of the hydroxide, and subscript "c" for the atoms of the carboxylate groups.

isotherms, compared to the values derived in other works on the similar systems MIL-47 (V) ${ }^{8}$ and MIL-53 (Al) ${ }^{44}$ and which are more than twice larger. Work is currently under progress to shed light on these differences.

\section{Adsorption in $\mathrm{Al}(\mathrm{OH})(1,4-n d c)$ and methylated systems}

Water adsorption in $\mathrm{Al}(\mathrm{OH})(1,4-\mathrm{ndc})$ follows a type- $\mathrm{V}$ adsorption isotherm, with a transition pressure around $1 \mathrm{kPa}$. This shape of isotherm is linked to the hydrophobic nature of the internal surface of the nanopores: because the water-MOF interactions are much smaller than the water-water interactions, the adsorption process is unfavourable from a free energy point of view at low pressure. The plateau of the isotherm, as seen in Fig. 3, corresponds to 18 water molecules per unit cell (around $3 \mathrm{kPa}$ ). These water molecules are all located in the larger channels of the material, filling them completely. Since each unit cell features two large channels, there are $\sim 9$ molecules per channel (of size $6.6 \AA$ along the $c$-axis) $\dagger$ These molecules form a small cylinder of water with a loosely ordered liquid-like structure, as was previously observed for water nanodroplets in spheroidal cages of hydrophobic zeolites. ${ }^{40}$ We also observe that each channel of the simulation box is always either empty or saturated with water. No intermediate filling state of the channel has been observed, except for transient intervals when a pore gets filled or emptied.

We also found that at very high pressure (above $100 \mathrm{MPa}$ ), water can be adsorbed into the small channels, forming a one-dimensional water chain corresponding to roughly 2 water molecules per channel. This kind of liquid water intrusion in extremely narrow, hydrophobic pores happening at high pressure has been thoroughly studied in zeolites. ${ }^{23,24,26}$ In the following discussion, we focus on adsorption of liquid-like water in the larger channels.

Fig. 4 displays the adsorption and desorption isotherms of water obtained by GCMC simulations of three methylated derivatives of $\mathrm{Al}(\mathrm{OH})(1,4-\mathrm{ndc})$, with varying degree of functionalisation: 2, 4 and $8-\mathrm{CH}_{3}$ groups per large channel, out of a total of 8 methylation sites. From these isotherms, it can be clearly seen that functionalisation of the material by methyl groups has an effect on four key properties of the isotherms: saturation uptake, adsorption pressure, steepness

$\dagger$ The discussion of adsorbed quantities in ref. 11 mistakenly features quantities per channel as "molecules per unit cell". All the data presented here and extracted from ref. 11 was converted directly from the results given in $\mathrm{cm}^{3} \mathrm{~g}^{-1}$ (STP).

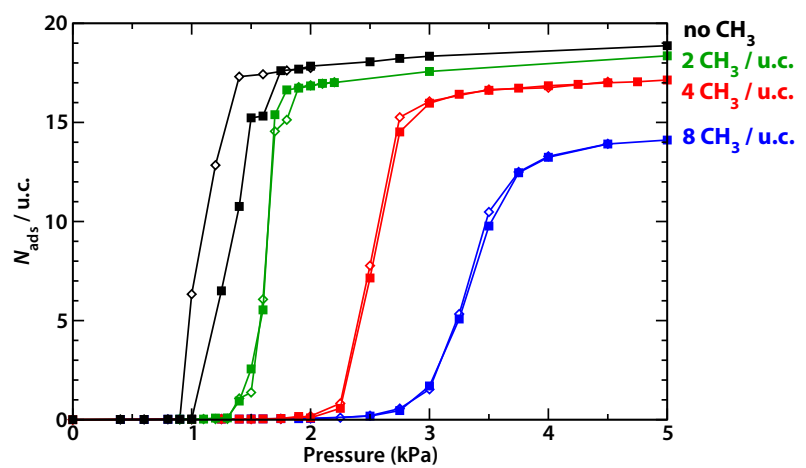

Fig. 4 Isotherms for adsorption (filled symbols) and desorption (open symbols) of water in $\mathrm{Al}(\mathrm{OH})(1,4-\mathrm{ndc})$ and derivatives of varying functionalisation proportion: original material (black), $2 \mathrm{CH}_{3}$ groups per channel (green), 4 groups (red) and 8 groups (fully substituted, blue).

of the isotherm and presence of hysteresis. The impact of methylation on saturation uptake can be simply explained as a steric effect, the addition of methyl groups reducing the cross-section (and thus the volume) of the pores.

Next, we turn to the influence of functionalisation on the adsorption pressure. As methyl groups are added, the pressure of the step of the isotherm shifts from $1.2 \mathrm{kPa}$ (for the unmodified material) to higher pressure: 1.6, 2.5 and $3.4 \mathrm{kPa}$ for the materials with 2, 4 and 8 methyl groups per channel respectively. We ascribe this effect to the changes of the chemical nature of the internal surface of the nanopores: the apolar methyl groups introduced by the functionalisation shield from the adsorbate the more polar carboxylate groups (see Fig. 2). Thus, as more methyl groups are grafted on the naphthalene aromatic ring, the overall water-material interaction diminishes and the water adsorption is shifted to higher pressure. It is worth noting that, even with the fully methylated material, the adsorption still happens in the gas phase (for $P<P_{\text {sat }}$ ).

\section{Nature of the adsorption transition; phase diagram of the confined water}

In order to gain further insight into the nature of the type-V adsorption isotherm of water in $\mathrm{Al}(\mathrm{OH})(1,4-\mathrm{ndc})$, and understand the effect of functionalisation on the steepness and hysteresis of the isotherm, we need to analyse it from the point of view of liquid-vapour phase transition under confinement. On both the adsorption and desorption isotherms in the original material (Fig. 4), one can note the presence of intermediate points between the empty $\left(N_{\text {ads }} \sim 0\right)$ and fully filled $\left(N_{\mathrm{ads}} \sim 18 \mathrm{molec} /\right.$ u.c. $)$ states. These can correspond to two possible situations: either they represent a series of intermediate thermodynamic states, with some of the channels filled and some of the channels empty; or, they represent weighted averages of the two coexisting metastable states, where all pores are filled or all empty. In the first case, the process of adsorption is a continuous transition, while in the second case, it is a first-order phase transition. The histograms of channel filling, presented in Fig. 5 for pressures going from $1 \mathrm{kPa}$ to $1.75 \mathrm{kPa}$, hint in favour of the continuous transition; while each pore is either completely filled or empty, 


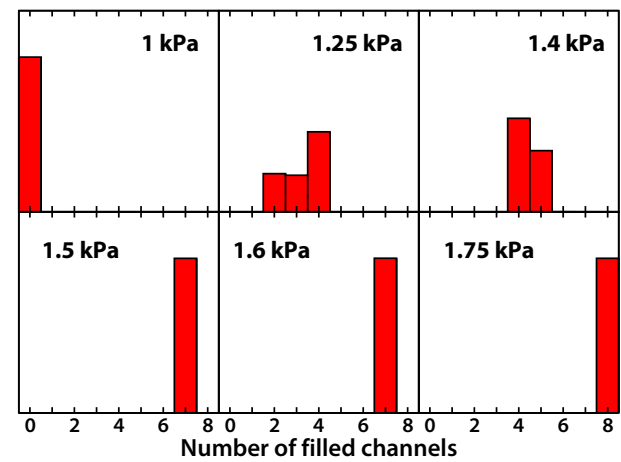

Fig. 5 Histograms of channel filling at different pressures, for water adsorption in $\mathrm{Al}(\mathrm{OH})(1,4-\mathrm{ndc})$.

a cooperative mechanism among neighbouring pores leads the global transition for the material to be continuous. This result, in particular, means that the study of this phenomenon is heavily dependent on the size of the chosen simulation box; initial GCMC simulations of a single unit cell of $\mathrm{Al}(\mathrm{OH})(1,4-\mathrm{ndc})$, not reported here, lead to the wrong conclusion that the adsorption follows a first-order phase transition, because the cooperativity between the pores is constrained by the periodic boundary conditions imposed.

In order to confirm this analysis, it is necessary to calculate the bidimensional free energy profiles $\Omega\left(N_{\mathrm{ads}}, \mu\right)$ of water sorption in the nanoporous material. In this study, we obtained this information using the Wang-Landau algorithm, as described in the "Computational Methods" section. The free energy profiles $\Omega\left(N_{\text {ads }}, \mu\right)$ of water in $\mathrm{Al}(\mathrm{OH})(1,4-\mathrm{ndc})$ are presented in Fig. 6 for chemical potentials of water corresponding to pressures in the range $1.0-1.7 \mathrm{kPa}$, which is the most relevant to the discussion. It can be seen, firstly, that each of these profiles present a number of local minima, corresponding to metastable states of the system. Visual inspection of the configurations of the system in each of those states showed that they all correspond to a certain number of channels filled in the $2 \times 2 \times 3$ supercell simulated. Secondly, it is to be noted that, upon increase of the water pressure, the relative stability of these states varies, and the most stable state accordingly shifts from its initial value of $N_{\mathrm{ads}} \approx 0$ to values of $N_{\text {ads }} \approx 2.5,4.5,6.5,11.0,13$ and 18 , which correspond respectively to $N_{\text {filled }}=1,2,3,5,6$ and 8 channels filled (of a total of 8 channels in the supercell). Thirdly, the free energy barriers present between these metastable states range between $\sim 10 k T$, for the smallest, and $15 k T$ for the highest barrier, separating the $N_{\text {filled }}=0$ and $N_{\text {filled }}=1$ states. From these free energy profiles, we can also calculate the equilibrium adsorption pressure as the water pressure for which the states $N_{\text {filled }}=0$ and $N_{\text {filled }}=8$ have the same free energy. The value of that pressure is $P_{\mathrm{eq}} \approx 1.2 \mathrm{kPa}$. This is in agreement with the calculated adsorption and desorption isotherms, as it falls squarely in the middle of the hysteresis loop.

To summarise, the free energy profiles confirm the insight given by the histograms of channel filling for adsorption (and the similar ones obtained for desorption) that the adsorption and desorption transitions proceed through intermediate metastable states. The number of these

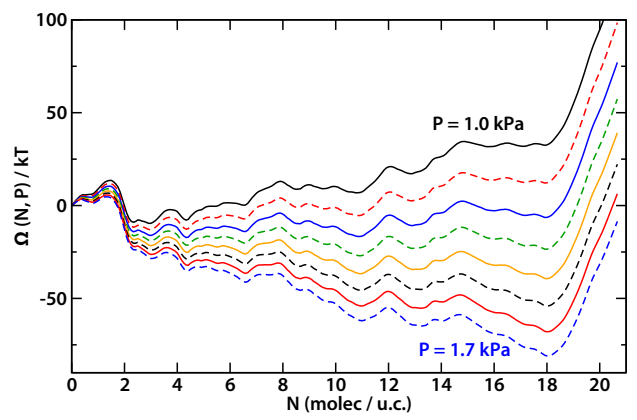

Fig. 6 Profiles of free energy $\Omega(\mu, N) / k T$ as a function of water loading of the unit cell $N$, for chemical potentials corresponding to pressures in the range $1-1.7 \mathrm{kPa}$ (with a $0.1 \mathrm{kPa}$ increment between curves), for the non-substituted $\mathrm{Al}(\mathrm{OH})(1,4-\mathrm{ndc})$ material.

intermediate states is linked to the number of channels in the system simulated, and can be characterised overall as a continuous transition in the limit of large system size, since the number of intermediate states then becomes infinite. However, this continuous nature of the transition coexists with the presence of type- $\mathrm{V}$ isotherms and a hysteresis loop, which are more often attributed to first-order phase transitions. $^{24}$ The reason for this hysteresis is found in the existence of the intermediate states and the free energy barriers, some much larger than $k T$, in between these states.

For the methyl-functionalised materials, the same continuous nature of the adsorption transition is observed. It is also remarkable that, with increasing number of methyl groups, the steepness of the isotherm diminishes, a sign that the transition is smoother and happens over a larger range of pressure. This is confirmed by the histograms of adsorbed water quantities in the fully-methylated system, at various pressure, which are shown in Fig. 7. The multimodal nature of the histograms confirms that, even in this material, each pore is still either fully filled or empty. Furthermore, the number of "states" involved at intermediate pressures is larger than in the original material; at $3.25 \mathrm{kPa}$, for example, the number of pores filled can vary between 0 and 6 (inclusively). From the continuous, equilibrated $\pi(N)$ histograms, we calculated the profiles of free energy as a function of water loading, as $\Omega(\mu, N)=-k T \log \pi(\mu, N) . \ddagger$ These free energy profiles are presented in Fig. 8, and they show a marked difference with the profiles of the nonsubstituted material (Fig. 6). While they still show intermediate minima corresponding to the metastable states already discussed above (at lower water loading, due to the diminished porous volume), both the free energy differences between the minima and the free energy barriers between them are of a much smaller magnitude (in the 0-5 $k T$ range). This is in keeping with, and explains, the absence of hysteresis in the adsorption-desorption isotherms of the methylated $\mathrm{Al}(\mathrm{OH})(1,4-\mathrm{ndc})$ materials. We attribute the smoother free energy profiles of these systems to

$\ddagger$ While this direct calculation from the GCMC simulations was not possible for the non-substituted $\mathrm{Al}(\mathrm{OH})(1,4-\mathrm{ndc})$ material, because of the presence of hysteresis loops indicating that the thermodynamic equilibrium was not reached during the time of our simulations, the simulations of adsorption in the methylated materials appear fully equilibrated. 


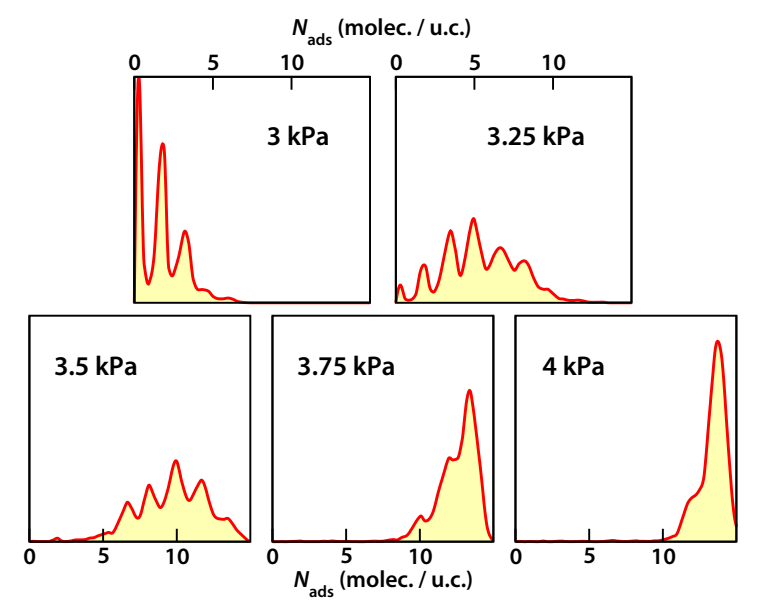

Fig. 7 Histograms of adsorbed water quantities at different pressures, for water adsorption in the fully methylated system.

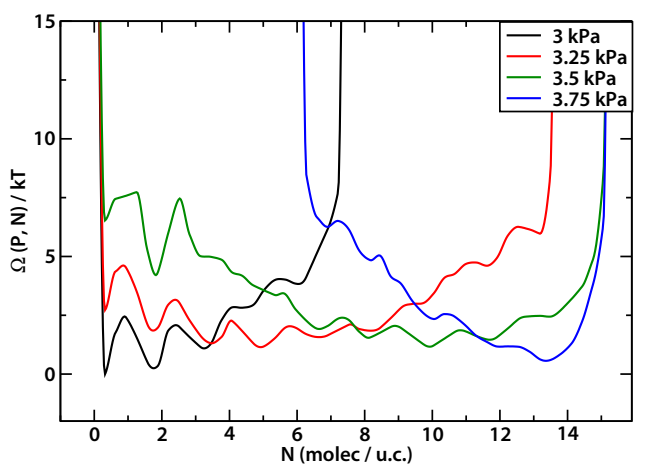

Fig. 8 Profiles of free energy $\Omega(\mu, N) / k T$ as a function of water loading of the unit cell $N$, for pressures in the range $3-3.75 \mathrm{kPa}$, for the fully substituted material.

the fact that the $-\mathrm{CH}_{3}$ groups on the surface of the pore hide the heterogeneity of the underlying groups, composed of the $\mathrm{Al}(\mathrm{OH})$ chains and the naphthalene rings.

In order to explain the decreased smoothness of the isotherms when the functionalisation ratio is increased, as well as rationalise the results presented so far, we propose a schematic family of phase diagrams for water confined in $\mathrm{Al}(\mathrm{OH})(1,4-\mathrm{ndc})$ and its methylated derivatives. We focus here on the liquid-vapour transition, leaving the liquid-solid and solid-vapour branches of the diagram voluntarily unspecified. This phase diagram is based on the four following results: (i) water adsorption in $\mathrm{Al}(\mathrm{OH})(1,4-\mathrm{ndc})$ happens at pressure lower than $P_{\text {sat }}$; (ii) the adsorption transition is continuous, meaning the confined water is supercritical at $298 \mathrm{~K}$; (iii) adding methyl groups increases the adsorption pressure; (iv) as confinement increases, the critical point shifts to lower temperature. ${ }^{45}$ The diagram obtained, represented in Fig. 9, is markedly different from that inferred for hydrophobic zeolites, where adsorption happens at $P>P_{\text {sat }}$.

This diagram allows us to explain the decreased steepness of the adsorption isotherms as methyl groups are added to the internal surface of the pore. If one draws a vertical line corresponding to the temperature of $298 \mathrm{~K}$, one can observe that, due to the features of this phase diagram, as more methyl groups are present in the material, the adsorption happens

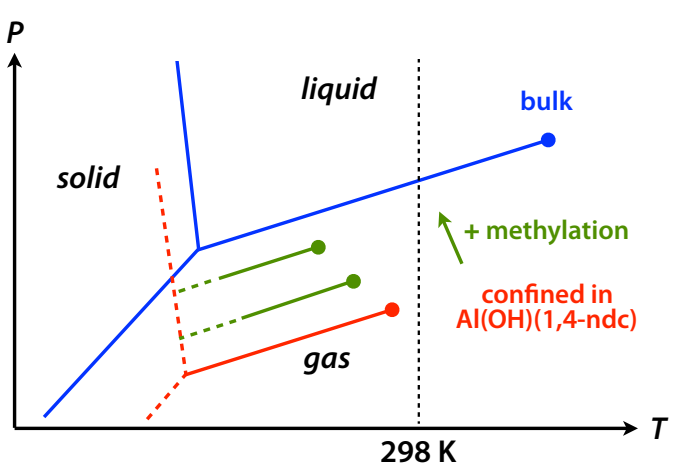

Fig. 9 Schematic liquid-vapour part of the phase diagram for water confined in $\mathrm{Al}(\mathrm{OH})(1,4-\mathrm{ndc})$ (in red) and methyl-substituted derivatives (in green, with increasing functionalisation upwards), compared to bulk water (in blue).

further and further away from the critical point of the confined water. The transition is thus expected to be "more continuous", and the isotherm smoother, as we move away from the critical point, as the compressibility of the supercritical fluid diminishes.

Finally, we have made attempts to obtain direct quantitative information about this phase diagram of confined water by performing GCMC simulations of water adsorption at lower temperature, in the hope of seeing a first-order phase transition in the nonfunctionalised material. We have not, so far, been able to observe this phenomenon in the range 275-298 K, leading to the conclusion that the critical point of water in $\mathrm{Al}(\mathrm{OH})(1,4-\mathrm{ndc})$ is located at $T<275 \mathrm{~K}$.

\section{Conclusions}

We used grand canonical Monte Carlo simulations to investigate the adsorption of water in the hydrophobic metalorganic framework $\mathrm{Al}(\mathrm{OH})(1,4$-naphthalenedicarboxylate). After validating a set of partial charges for the material against the experimentally available isotherm, we studied the influence of methyl substitution of the nanopores' internal surface on the adsorption isotherms. We determined the nature of the adsorption transition, showing it to be of continuous nature in all cases. We rationalised the shape and steepness of the isotherms, as well as the evolution of hysteresis loops, in terms of a phase diagram for water confined in this family of materials. Work is in progress studying the broader question of forcefields for the description of adsorption of very polar molecules (such as water in metal-organic frameworks), by comparing the atomic partial charges adjusted in this work to reproduce experimental results with partial charges determined by other methods (including ab initio calculations), both on $\mathrm{Al}(\mathrm{OH})(1,4-\mathrm{ndc})$ and other MOFs.

\section{Acknowledgements}

This work was supported by the French Agence Nationale de la Recherche, under contracts ANR-06-CO2-007 and BLANC06-3_144027. The authors thank Anne Boutin and Randy Snurr for fruitful discussions, and Jean-Marie Teuler for the parallelised Wang-Landau simulation code. 


\section{References}

1 J.-R. Li, R. J. Kuppler and H.-C. Zhou, Chem. Soc. Rev., 2009, 38, $1477-1504$.

2 M. Dincă and J. R. Long, Angew. Chem., Int. Ed., 2008, 47, 6766-6779.

3 P. Küsgens, M. Rose, I. Senkovska, H. Fröde, A. Henschel, Sven Siegle and S. Kaskel, Microporous Mesoporous Mater., 2009, 120, 325-330.

4 J. A. Greathouse and M. D. Allendorf, J. Am. Chem. Soc., 2006, 128, 10678-10679.

5 A. Kondo, T. Daimaru, H. Noguchi, T. Ohba, K. Kaneko and H. Kanoh, J. Colloid Interface Sci., 2007, 314, 422-426.

6 Y. Li and R. T. Yang, Langmuir, 2007, 23, 12937-12944.

7 J. Y. Lee, D. H. Olson, L. Pan, T. J. Emge and J. Li, Adv. Funct. Mater., 2007, 17, 1255-1262.

8 J. M. Castillo, T. J. H. Vlugt and S. Calero, J. Phys. Chem. C, 2008, 112, 15934-15939.

9 A. Ö. Yazaydın, A. I. Benin, S. A. Faheem, P. Jakubczak, J. J. Low, R. R. Willis and R. Q. Snurr, Chem. Mater., 2009, 21, $1425-1430$.

10 J. J. Low, A. I. Benin, P. Jakubczak, J. F. Abrahamian, S. A. Faheem and R. R. Willis, J. Am. Chem. Soc., 2009, 131, 15834-15842.

11 A. Comotti, S. Bracco, P. Sozzani, S. Horike, R. Matsuda, J. Chen, M. Takata, Y. Kubota and S. Kitagawa, J. Am. Chem. Soc., 2008, 130, 13664-13672.

12 J.-C. Moïse, J.-P. Bellat and A. Méthivier, Microporous Mesoporous Mater., 2001, 43, 91-101.

13 N. Giovambattista, P. J. Rossky and P. G. Debenedetti, Phys. Rev. E: Stat., Nonlinear, Soft Matter Phys., 2006, 73, 041604.

14 N. Desbiens, A. Boutin and I. Demachy, J. Phys. Chem. B, 2005, 109, 24071-24076.

15 C. E. Ramachandran, S. Chempath, L. J. Broadbelt and R. Q. Snurr, Microporous Mesoporous Mater., 2006, 90, 293-298.

16 H. Nishihara, Y. Fukura, K. Inde, K. Tsuji, M. Takeuchi and T. Kyotani, Carbon, 2008, 46, 48-53.

17 W. Kauzmann, Adv. Protein Chem., 1959, 14, 1-63.

18 K. A. Dill, Biochemistry, 1990, 29, 7133-7155.

19 D. Chandler, Nature, 2002, 417, 491-491.

20 C. L. Brooks III, J. N. Onuchic and D. J. Wales, Science, 2001, 293, 612-613.

21 N. Giovambattista, P. G. Debenedetti and P. J. Rossky, J. Phys. Chem. B, 2007, 111, 9581-9587.

22 M. Szöri, D. J. Tobias and M. Roeselová, J. Phys. Chem. B, 2009, 113, 4161-4169.

23 F. Cailliez, M. Trzpit, M. Soulard, I. Demachy, A. Boutin, J. Patarin and A. H. Fuchs, Phys. Chem. Chem. Phys., 2008, 10, 4817-4826.
24 F. Cailliez, A. Boutin, I. Demachy and A. H. Fuchs, Mol. Simul., 2009, 35, 24-30.

25 F.-X. Coudert, F. Cailliez, R. Vuilleuinier, A. H. Fuchs and A. Boutin, Faraday Discuss., 2009, 141, 377-398.

26 N. Desbiens, A. Boutin and I. Demachy, J. Phys. Chem. B, 2005, 109, 24071-24076.

27 A. D. Mackie, B. Tavitian, A. Boutin and A. H. Fuchs, Mol. Simul., 1997, 19, 1-15.

28 R. F. Cracknell, D. Nicholson, N. G. Parsonage and H. Evans, Mol. Phys., 1990, 71, 931-943.

29 V. Lachet, A. Boutin, B. Tavitian and A. H. Fuchs, J. Phys. Chem. $B, 1998,102,9224-9233$.

30 A. Di Lella, N. Desbiens, A. Boutin, I. Demachy, P. Ungerer, J.-P. Bellat and A. H. Fuchs, Phys. Chem. Chem. Phys., 2006, 8, 5396-5406.

31 E. Kim, R. Faller, Q. Yan, N. Abbott and J. de Pablo, J. Chem. Phys., 2002, 117, 7781-7787.

32 M. Chopra, M. Müller and J. de Pablo, J. Chem. Phys., 2006, 124, 134102.

33 F. Wang and D. Landau, Phys. Rev. E: Stat., Nonlinear, Soft Matter Phys., 2001, 64, 056101.

34 F. Wang and D. Landau, Phys. Rev. Lett., 2001, 86, 2050-2053.

35 M. Shell, P. Debenedetti and A. Panagiotopoulos, Phys. Rev. E: Stat., Nonlinear, Soft Matter Phys., 2002, 66, 056703.

36 H. W. Horn, W. C. Swope, J. W. Pitera, J. D. Madura, T. J. Dick, G. L. Hura and T. Head-Gordon, J. Chem. Phys., 2004, 120, 9665.

37 H. W. Horn, W. C. Swope and J. W. Pitera, J. Chem. Phys., 2005, 123, 194504.

38 S. L. Mayo, B. D. Olafson and W. A. Goddard, J. Phys. Chem., 1990, 94, 8897.

39 A. K. Rappe, C. J. Casewit, K. S. Colwell, W. A. Goddard and W. M. Skiff, J. Am. Chem. Soc., 1992, 114, 10024.

40 F.-X. Coudert, R. Vuilleumier and A. Boutin, ChemPhysChem, 2006, 7, 2464-2467.

41 N. Desbiens, I. Demachy, A. H. Fuchs, H. Kirsch-Rodeschini, M. Soulard and J. Patarin, Angew. Chem., Int. Ed., 2005, 44, 5310-5313.

42 V. Finsy, S. Calero, E. García-Pérez, P. J. Merkling, G. Vedts, D. E. De Vos, G. V. Baron and J. F. M. Denayer, Phys. Chem. Chem. Phys., 2009, 11, 3515-3521.

43 K. S. W. Sing, D. H. Everett, R. A. W. Haul, L. Moscou, R. A. Pierotti, J. Rouquerol and T. Siemieniewska, Pure Appl. Chem., 1985, 57, 603-619.

44 N. A. Ramsahye, G. Maurin, S. Bourrelly, P. L. Llewellyn, T. Devic, C. Serre, T. Loiseau and G. Férey, Adsorption, 2007, 13, 461-467.

45 I. Brovchenko, A. Geiger and A. Oleinikova, J. Chem. Phys., 2004, 120, 1958 